\title{
Management of pregnancy at and beyond 41 completed weeks of gestation in low- risk women: a secondary analysis of two WHO multi-country surveys on maternal and newborn health
}

Kyaw Swa Mya ${ }^{1,2}$, Malinee Laopaiboon', Joshua P. Vogel ${ }^{3,4}$, Jose Guilherme Cecatti ${ }^{5}$, João Paulo Souza ${ }^{3,4,6}$, Ahmet Metin Gulmezoglu ${ }^{3,4}$, Eduardo Ortiz-Panozo ${ }^{7}$, Suneeta Mittal ${ }^{8}$, Pisake Lumbiganon ${ }^{9^{*}}$ (i) and on behalf of the WHO multi-country survey on maternal and newborn health research network

\begin{abstract}
Background: The World Health Organization (WHO) recommends induction of labour (IOL) for women who have reached 41 completed weeks of pregnancy without spontaneous onset of labour. Many women with prolonged pregnancy and/or their clinicians elect not to induce, and chose either elective caesarean section (ECS) or expectant management (EM). This study intended to assess pregnancy outcomes of IOL, ECS and EM at and beyond 41 completed weeks.

Methods: This study is a secondary analysis of the WHO Global Survey (WHOGS) and the WHO Multi-country Survey (WHOMCS) conducted in Africa, Asia, Latin America and the Middle East. There were 33,003 women with low risk singleton pregnancies at $\geq 41$ completed weeks from 292 facilities in 21 countries. Multilevel logistic regression model was used to assess associations of different management groups with each pregnancy outcome accounted for hierarchical survey design. The results were presented by adjusted odds ratios (aORs) with 95\% confidence intervals (Cls) after adjusting for age, education, marital status, parity, previous caesarean section (CS), birth weight, and facility capacity index score.
\end{abstract}

Results: The prevalence of prolonged pregnancy at facility setting in WHOGS, WHOMCS and combined databases were $7.9 \%, 7.5 \%$ and $7.7 \%$ respectively. Regarding to maternal adverse outcomes, EM was significantly associated with decreased risk of CS rate consistently in both databases i.e. (aOR0.76; 95\% Cl: 0.66-0.87) in WHOGS, (aOR0.67; 95\% Cl: 0.59-0.76) in WHOMCS and (aOR0.70; 95\% Cl: 0.64-0.77) in combined database, compared to IOL. Regarding the adverse perinatal outcomes, ECS was significantly associated with increased risks of neonatal intensive care unit admission (aOR1.76; 95\% Cl: 1.28-2.42) in WHOMCS and (aOR1.51; 95\% Cl: 1.19-1.92) in combined database compared to IOL but not significant in WHOGS database.

(Continued on next page)

\footnotetext{
* Correspondence: pisake@kku.ac.th

${ }^{9}$ Department of Obstetrics and Gynaecology, Faculty of Medicine, Khon Kaen

University, Khon Kaen 40002, Thailand

Full list of author information is available at the end of the article
} 
(Continued from previous page)

Conclusions: Compared to IOL, ECS significantly increased risk of NICU admission while EM was significantly associated with decreased risk of CS. ECS should not be recommended for women at 41 completed weeks of pregnancy. However, the choice between IOL and EM should be cautiously considered since the available evidences are still quite limited.

Keywords: Prolonged pregnancy, Post-term pregnancy, Induction of labour, Elective caesarean section, Expectant management, Pregnancy outcomes

\section{Plain English summary}

Pregnancies beyond 41 completed weeks are associated with adverse outcomes. Hence, the World Health Organization recommends inducing labour for women who have reached 41 completed weeks of pregnancy without spontaneous labour pain. Many of such women and/ or their clinicians do not want to induce, instead they prefer to deliver by caesarean section or expectant management (awaiting spontaneous onset of labour).

We compared pregnancy outcomes of women at 41 completed weeks of pregnancy by three different managements - induction of labour, elective caesarean section and expectant management using two large, WHO databases conducted in Africa, Asia, Latin America and the Middle East. We did not find any difference in adverse pregnancy outcomes between induction group and expectant group except higher caesarean section rate in induction of labour group.

We found that neonatal intensive care unit admission was higher in newborns delivered by elective caesarean section compared to that of induction of labour. Our findings showed that elective caesarean section had increased risk of adverse neonatal outcomes and should not be recommended. However, the choice between induction of labour and expectant management should be cautiously considered since the available evidences are still quite limited.

\section{Background}

The World Health Organization (WHO) recommends induction of labour (IOL) for women who have reached 41 completed weeks of pregnancy without spontaneous onset of labour [1]. Rates of IOL vary across countries. IOL rates for high-income countries were $23.4 \%$ of deliveries in United States in 2010 [2], 22.1\% of deliveries in England between 2011 and 2012 [3] and 25.4\% of deliveries in Australia in 2010 [4]. The rates also vary for low and middle-income countries (LMIC). The WHO Global Survey on Maternal and Perinatal Health reported the prevalence of IOL in facility deliveries as $4.4 \%$ in seven African countries, $12.1 \%$ in nine Asian countries [5] and $11.4 \%$ in eight Latin American countries [6]. IOL is specifically recommended to prevent complications of prolonged pregnancy, such as increased perinatal mortality, stillbirth, fetal growth restriction, meconium aspiration syndrome and macrosomia [7-10]. However, IOL itself carries the risk of uterine hyperstimulation, increased instrumental delivery, uterine rupture, fetal distress and Caesarean section (CS) [1].

Many women with prolonged pregnancy ( $\geq 41$ weeks) and/or their clinicians often elect not to induce, and chose either ECS or expectant management EM (awaiting spontaneous onset of labour). The reasons for choosing CS may be not only to manage the prolonged pregnancy, but also be the preferred mode of delivery for the women and/ or the clinicians [11].

Systematic reviews of randomized controlled trials have compared the risks and benefits of IOL compared to EM at and beyond 41 weeks gestation [12-16]. All these systematic reviews assessed perinatal death and CS rate as primary outcomes, and other maternal and perinatal morbidities such as postpartum haemorrhage, ruptured uterus, meconium aspiration, Apgar score, NICU admission, stillbirth and early neonatal death as secondary outcomes. These reviews consistently reported that IOL at 41 completed weeks of gestation reduced the complications of postterm pregnancies compared to EM. However, the risks and benefits of IOL compared to ECS for women with prolonged pregnancy have not been as thoroughly explored. Furthermore, analysis of clinical data can provide insight into the effectiveness of interventions in "real life" settings. This analysis aimed to explore not only the risks and benefits of IOL but also that of ECS regarding to pregnancy outcomes among women with prolonged pregnancy in two large multi-country databases of facility deliveries in predominantly low- and middle-income countries.

\section{Methods \\ Study design and setting}

This study is a secondary analysis of two WHO databases: the WHO Global Survey (WHOGS) on Maternal and Perinatal Health [17] conducted in Africa, Asia and Latin America and the WHO Multi-country Survey (WHOMCS) on Maternal and Newborn Health conducted in Africa, Asia, Latin America and the Middle East [18]. WHOGS was conducted to explore the 
association between CS and maternal and perinatal outcomes in 286,565 women giving birth in 373 facilities in 24 countries during 2004-2008. Building on the existing WHOGS network, the WHOMCS aimed to assess severe maternal and perinatal morbidity using the WHO maternal near-miss criteria in 314,623 women at 359 facilities in 29 countries during 2010-2011. All the participating countries except Japan and Qatar were in low and middle-income category (according to World Bank classifications) [19].

Details of the survey methods have been published elsewhere [17-20]. In brief, they were multi-centre, facility based, cross-sectional studies which used a stratified multistage cluster sampling method to select a sample of countries, provinces and health facilities. For the WHOGS, fourteen sub-regions from the six WHO regions were identified as the sampling frame. From each sub-region, four countries were randomly selected (probability proportional to population size). From each country, two provinces in addition to capital city were randomly selected. From each province, seven health facilities were randomly chosen from the health facilities with more than 1000 births per year and having ability to perform CS. In the WHOMCS, all WHOGS countries were invited to participate, however only 22 countries were able to participate. Two countries (Cuba and Algeria) were unable to participate. Within the remaining 22 countries, 32 facilities with very poor recruitment, data quality issues, or that were unable to participate were not included in the WHOMCS. Seven new countries (Afghanistan, Pakistan, Occupied Palestinian Territories, Mongolia, Jordan, Qatar and Lebanon) were added to improve global representation, bringing the total to 29 countries in WHOMCS.

Data in both surveys were collected using individual and institutional case record forms. Data were captured from the time the women first attended at facility for delivery until death, discharge or seventh postpartum day (whichever occurred first). Adverse pregnancy outcomes that occurred after discharge, during referral or after seventh postpartum day were not recorded. Data collection period was two months in health facilities with at least 6000 deliveries per year and three months in health facilities with less than 6000 deliveries per year. Socio-demographic characteristics, obstetric history, mode of delivery, labour characteristics and maternal and perinatal outcomes were collected for all women using pre-tested case record forms by trained data collectors. Health facility data concerning available obstetric and newborn services were also recorded in pre-tested institutional forms after consulting the head of department of obstetrics. Web-based data management systems were used for data entry of both databases. Internal consistency of data was randomly crosschecked comparing collected data and hospital records.
The technical content of both protocols was reviewed by specialist panels at the UNDP/UNFPA/UNICEF/ WHO/ World Bank Special Programme of Research, Development and Research Training in Human Reproduction. The Specialist Panel on Epidemiological Research reviewed and approved the WHOGS study protocol for technical content; the Research Project Review Panel (name of panel was changed in 2010) reviewed and approved the technical content of the WHOMCS

The WHOGS and the WHOMCS addressed different primary research questions, but both studies used a common list of core variables that enabled this secondary analysis. Some countries and facilities did not participate in both surveys; therefore, we restricted our analysis to the facilities and the countries that contributed data to both databases. Under this restriction, Cuba and Algeria from WHOGS and Afghanistan, Lebanon, Mongolia, Occupied Palestinian Territory, Pakistan, Qatar and Jordan from WHOMCS were excluded. Angola was excluded from this analysis due to concerns regarding poor data quality for gestational age (a critical variable for this analysis). A total of 21 countries participated in this analysis. Of these 21 countries 26 facilities from WHOGS and 6 facilities from WHOMCS were not included in both surveys, hence, excluded in this analysis. In addition, the facilities with unreliable information on gestational age distribution (i.e., facilities in which gestational age missing more than $5 \%$, more than $70 \%$ of all deliveries occurred at a specific week, or where more than $30 \%$ or less than $1 \%$ of all deliveries were preterm) and with less than 100 total deliveries were excluded. Details on exclusion of women, facilities and countries are presented in Fig. 1. The current analysis has an analytical approach where three different procedures for managing low risk pregnancies at 41 completed weeks were evaluated in the same setting of 292 facilities in 21 countries.

\section{Study population}

At the individual level, women with GA $<41$ weeks or missing data were excluded. A total of 18,331 women from 233 facilities of WHOGS and 18,312 women from 235 facilities of WHOMCS were identified as prolonged pregnancies (women with GA $\geq 41$ completed weeks of gestation). From this cohort of pregnant women, those with two or more previous CS, abnormal fetal presentation, pregnancy complications (preeclampsia, eclampsia), associated systemic diseases (heart disease, lung disease, renal disease, HIV/AIDS, malaria/dengue, severe anaemia $(\mathrm{Hb}<7 \mathrm{~g} / \mathrm{L})$, and chronic hypertension) and women who delivered multiple births, babies with congenital malformation or macerated stillbirths were excluded. We excluded macerated stillbirths because 


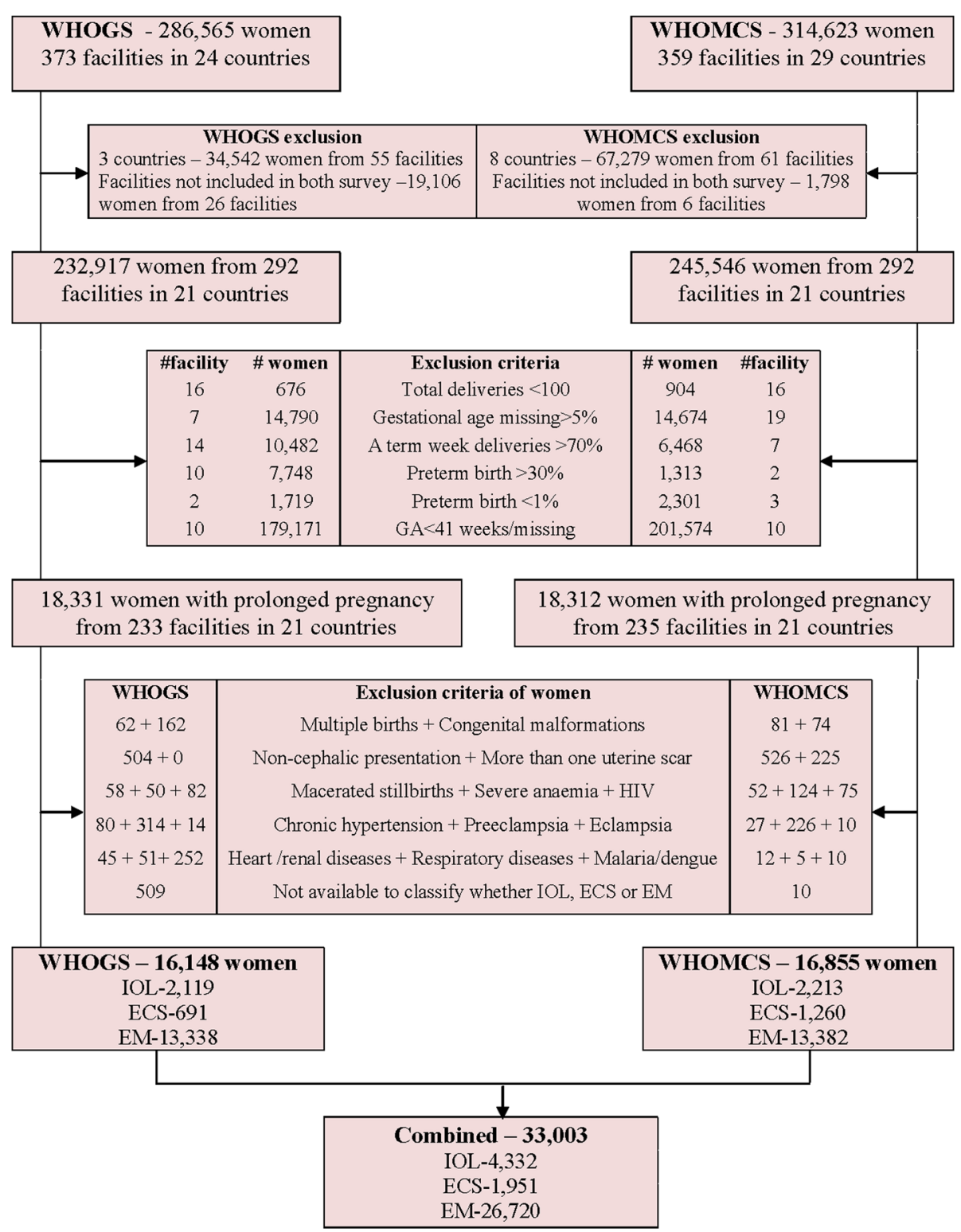

Fig. 1 View of the scree parallel plot and scree simulation for determining the number of factors to retain

they occurred before and were not consequences of IOL. Details are shown in Fig. 1.

In both surveys, we classified women using consistent definitions. Eligible women were classified into three groups based on the management at and beyond 41 completed weeks of gestation. Women who delivered their babies following IOL at 41 completed weeks were considered as the IOL group. Women of IOL group could deliver vaginally or by CS depending on whether the induction was successful or not. Women who delivered by ECS at 41 completed weeks in WHOGS and women delivered by prelabour CS at 41 completed weeks in WHOMCS were considered as ECS group. Women who delivered by spontaneous onset of labour at 41completed weeks and all women who delivered beyond 41 completed weeks (regardless of final mode of delivery) were considered as EM group. In this study, vaginal delivery included both normal and instrumental deliveries.

\section{Pregnancy outcomes and other variables}

We classified pregnancy outcomes into maternal and perinatal outcomes. For maternal adverse outcomes we assessed postpartum haemorrhage ( $\mathrm{PPH})$, uterine rupture, admission to maternal ICU, maternal postpartum length of stay $>7$ days and severe maternal outcome. CS rate was also compared between IOL and EM groups. Postpartum haemorrhage, uterine rupture and severe 
maternal outcome were defined by different criteria for the two databases (as somewhat different variables were available on maternal outcomes). We presented these criteria for each database in Table 1.

Perinatal adverse outcomes included stillbirth, early neonatal death, perinatal death, Apgar scores less than 7 at $5 \mathrm{~min}$ and admission to the neonatal intensive care unit (NICU).

Potential confounding factors were considered at both individual and facility levels. At the individual level, these included maternal age, education, marital status, parity, previous CS and birth weight. At the facility level, the availability of maternal healthcare services was classified into different levels, called facility capacity index (FCI) score. FCI score was calculated by using basic and essential services available at that facility and slightly different between WHOGS [21] and WHOMCS (due to differences in the institutional form used in the surveys) [22]. For consistency, we used FCI score calculated from WHOMCS in our analysis. It was ranged from 12 for the least resource service facility to 57 for the highest resource service facility. FCI score was used as a continuous variable and adjusted as a facility level covariate.

\section{Statistical analysis}

We used frequency to describe maternal and neonatal characteristics of the three sub-populations for analysis in both surveys. Comparisons of maternal and neonatal characteristics among different management groups were done using chi-square test. Multilevel logistic regression model was used to assess associations of different management groups with each pregnancy outcome, adjusted for the potential confounding factors and hierarchical survey design, using two levels (individual and facility level) for separate analysis of WHOGS and WHOMCS. For the combined database, source of data (WHOGS and WHOMCS) was used as an additional level. Risks of maternal and neonatal adverse outcomes associated with each management group were assessed separately for WHOGS, WHOMCS and the combined database to identify the any consistency of associations. The results were presented by crude and adjusted odds ratios (crude ORs and AORs) with corresponding 95\% confidence intervals (95\% CIs). IOL group was used as a reference group because this is the procedure currently recommended by WHO [1]. Statistical analyses were done using lme4 package [23] in R software [24].

\section{Results}

The prevalence of prolonged pregnancy at facility setting in WHOGS, WHOMCS and combined databases were $7.9 \%(18,331 / 232917), 7.5 \%(18,312 / 245546)$ and $7.7 \%$ (36,643/478463), respectively.

A total of 33,003 singleton pregnant women $(16,148$ women from WHOGS and 16,855 women from WHOMCS) with prolonged pregnancy ( $\geq 41$ completed weeks) were included in this analysis. Median gestational age for this cohort was 41 and $95 \%$ CIs were $41-$ 43 weeks. Amongst them, 13.1\% $(n=4332)$ were delivered by IOL, $5.9 \%(n=1951)$ were delivered by elective or prelabour CS and $80.9 \%(n=26,720)$ were in EM group. Details are presented in Fig. 1.

The details of maternal and neonatal characteristics among the three different management groups for WHOGS and WHOMCS are presented in Table 2. We found that pregnant women were significantly different with respect to maternal age, education, parity, previous CS and newborns birth weight among the three different management groups in both WHOGS and WHOMCS but marital status was significantly different only in WHOGS.

\section{Adverse maternal outcomes among different management groups}

Table 3 showed comparison of adverse maternal outcomes among different management groups for WHOGS, WHOMCS and combined database, respectively. The association could not be assessed for ruptured uterus and ICU admission outcomes in WHOMCS as women with these outcomes were found only in EM group. In combined database, increased risk of ruptured uterus outcome was not statistically significant for EM compared to IOL (aOR 1.98; 95\% CI: 0.51-7.65). EM was significantly associated with decreased risk of CS rate consistently in both databases i.e. (aOR0.76; 95\% CI: 0.66-0.87) in WHOGS, (aOR0.67; 95\% CI: 0.59-0.76) in WHOMCS and (aOR0.70; 95\% CI: 0.64-0.77) in combined database, compared to IOL.

Table 1 Criteria used to define maternal outcomes in WHOGS and WHOMCS databases

\begin{tabular}{lll}
\hline Maternal Outcomes & WHOGS Database & WHOMCS Database \\
\hline Uterine rupture & Women who had laparotomy for uterine rupture or delivered & Women with complication of ruptured uterus \\
& by CS due to suspected/imminent uterine rupture & Women with complication of PPH \\
PPH & Women who had blood transfusion due to PPH or received & Wterotonics as a treatment of PPH \\
Severe maternal outcome & $\begin{array}{l}\text { Women who had experienced in any of death or severe } \\
\text { maternal morbidity - blood transfusion, hysterectomy, }\end{array}$ & Women who had experienced maternal death maternal near miss according to WHO criteria [20] \\
\hline
\end{tabular}


Table 2 Maternal and neonatal characteristics of three different management groups for WHOGS and WHOMCS databases

\begin{tabular}{|c|c|c|c|c|c|c|c|c|}
\hline \multirow[t]{2}{*}{ Variables } & \multicolumn{3}{|c|}{$\begin{array}{l}\text { WHOGS }(N=16,148) \\
\text { Different management groups }\end{array}$} & \multirow[t]{2}{*}{$P$ value } & \multicolumn{3}{|c|}{$\begin{array}{l}\text { WHOMCS }(N=16,855) \\
\text { Different management groups }\end{array}$} & \multirow[t]{2}{*}{$P$ value } \\
\hline & $\begin{array}{l}\mathrm{IOL} \\
(N=2119) \\
n(\%)\end{array}$ & $\begin{array}{l}\text { ECS } \\
(N=691) \\
n(\%)\end{array}$ & $\begin{array}{l}\text { EM } \\
(N=13,338) \\
n(\%)\end{array}$ & & $\begin{array}{l}\overline{\mathrm{IOL}} \\
(N=2213) \\
n(\%)\end{array}$ & $\begin{array}{l}\text { ECS } \\
(N=1260) \\
n(\%)\end{array}$ & $\begin{array}{l}\text { EM } \\
(N=13,382) \\
n(\%)\end{array}$ & \\
\hline \multicolumn{9}{|l|}{ Maternal characteristics } \\
\hline \multicolumn{9}{|l|}{ Age (Years) } \\
\hline$\leq 19$ & $168(7.9)$ & $53(7.7)$ & $1578(11.8)$ & $<0.001$ & $222(10.0)$ & $104(8.3)$ & $1532(11.5)$ & 0.003 \\
\hline $20-34$ & $1786(84.3)$ & $571(82.6)$ & $10,623(79.7)$ & & $1801(81.4)$ & $1035(82.1)$ & $10,606(79.3)$ & \\
\hline$\geq 35$ & $165(7.8)$ & $67(9.7)$ & $1137(8.5)$ & & $190(8.6)$ & $121(9.6)$ & $1244(9.3)$ & \\
\hline \multicolumn{9}{|c|}{ Education (School years) } \\
\hline$<7$ & $412(20.0)$ & $119(17.3)$ & 3218 (24.3) & $<0.001$ & $290(13.1)$ & $195(15.4)$ & $2854(21.3)$ & $<0.001$ \\
\hline $7-12$ & $1173(56.9)$ & $409(59.4)$ & $7842(59.3)$ & & $1151(52.0)$ & $728(57.8)$ & $7671(57.3)$ & \\
\hline$>12$ & $476(23.1)$ & $161(23.4)$ & $2176(16.4)$ & & $772(34.9)$ & $337(26.8)$ & $2857(21.4)$ & \\
\hline \multicolumn{9}{|l|}{ Marital } \\
\hline With partner & $1954(92.3)$ & $620(90.0)$ & $11,663(87.5)$ & $<0.001$ & $1944(87.8)$ & 1119 (88.8) & $11,812(88.3)$ & 0.692 \\
\hline Without partner & $164(7.7)$ & $69(10.0)$ & $1660(12.5)$ & & $269(12.2)$ & $141(11.2)$ & $1570(11.7)$ & \\
\hline \multicolumn{9}{|l|}{ Parity } \\
\hline Primiparous & $1223(57.7)$ & $372(53.8)$ & $6353(47.6)$ & $<0.001$ & $1304(59.0)$ & $797(63.3)$ & 6496 (48.6) & $<0.001$ \\
\hline Multiparous & $896(42.3)$ & $319(46.2)$ & $6985(52.4)$ & & $908(41.0)$ & $462(36.7)$ & $6879(51.4)$ & \\
\hline \multicolumn{9}{|l|}{ Previous CS } \\
\hline Yes & $43(2.0)$ & $171(24.7)$ & $731(5.5)$ & $<0.001$ & $75(3.4)$ & $259(20.7)$ & $879(6.6)$ & $<0.001$ \\
\hline No & $2076(98.0)$ & $520(75.3)$ & $12,607(94.5)$ & & 2135 (96.6) & $993(79.3)$ & $12,480(93.4)$ & \\
\hline \multicolumn{9}{|l|}{ Neonatal characteristics } \\
\hline \multicolumn{9}{|l|}{ Birth weight } \\
\hline$<2500 \mathrm{~g}$ & $57(2.7)$ & $9(1.3)$ & $359(2.7)$ & $<0.001$ & $71(3.2)$ & $33(2.6)$ & $448(3.4)$ & $<0.001$ \\
\hline $2500-4000 \mathrm{~g}$ & $1970(93.0)$ & $585(84.7)$ & $12,220(91.6)$ & & 2017 (91.1) & $1084(86.0)$ & $12,208(91.2)$ & \\
\hline$>4000 \mathrm{~g}$ & $92(4.3)$ & $97(14.0)$ & $758(5.7)$ & & $125(5.7)$ & $143(11.4)$ & $726(5.4)$ & \\
\hline
\end{tabular}

(IOL Induction of labour group, ECS Elective Caesarean section group, EM Expectant management group)

Adverse perinatal outcomes among different management groups

Use of ECS was significantly associated with increased risks of NICU admission, aOR 1.76; 95\%CI: $1.28-2.42$ in WHOMCS and aOR 1.51; 95\%CI: $1.19-1.92$ in combined database, compared to IOL. The association was not statistically significant in WHOGS database. Apart from NICU outcome, the rest of perinatal outcomes were not significantly associated with different managements groups. Details are presented in Table 4.

One consideration in this analysis is that some women in the EM group may experience induction and/or CS at a later gestational age. To account for this, we re-classified those women in the EM group (at 41 completed weeks) who experienced induction or ECS later in pregnancy (i.e. beyond 41 completed weeks). There were 1759 pregnant women in the EM group with deliveries at beyond 41 weeks of gestation. They could be classified to IOL group for 1217 women and ECS group for 542 women. We did sensitivity analyses of the associations of the new classification of management groups with each pregnancy outcome in the combined database. The results showed consistent findings with our main analysis. In addition the sensitivity analyzed results provided stronger associated with increased risk of postpartum length of stay $>7$ days outcome (aOR1.59; 95\% CI: 1.17-2.18) and admission to NICU outcome (aOR1.54; 95\% CI: 1.25-1.90) for ECS compared to IOL. The stronger associated with decreased risk of severe maternal outcome (aOR0.73; 95\% CI: 0.55-0.99), rate of CS (aOR0.65; 95\% CI: 0.59-0.70) and admission to NICU outcome (aOR0.84; 95\% CI: 0.72-0.97) were also seen for EM compared to IOL. Details are presented in Table 5. We also assessed the risk of stillbirths by week delivered using 41 weeks as a reference group. The risk was not significantly different at 42 weeks (aOR1.47; 95\% CI: 0.82-2.62). However, as gestational age reached 43,44 and 45 weeks, the risk of stillbirth were significantly increased, aOR3.45; 95\% CI: 1.32-9.03 for 43 weeks, aOR 6.15; 95\% CI: $1.38-27.48$ 


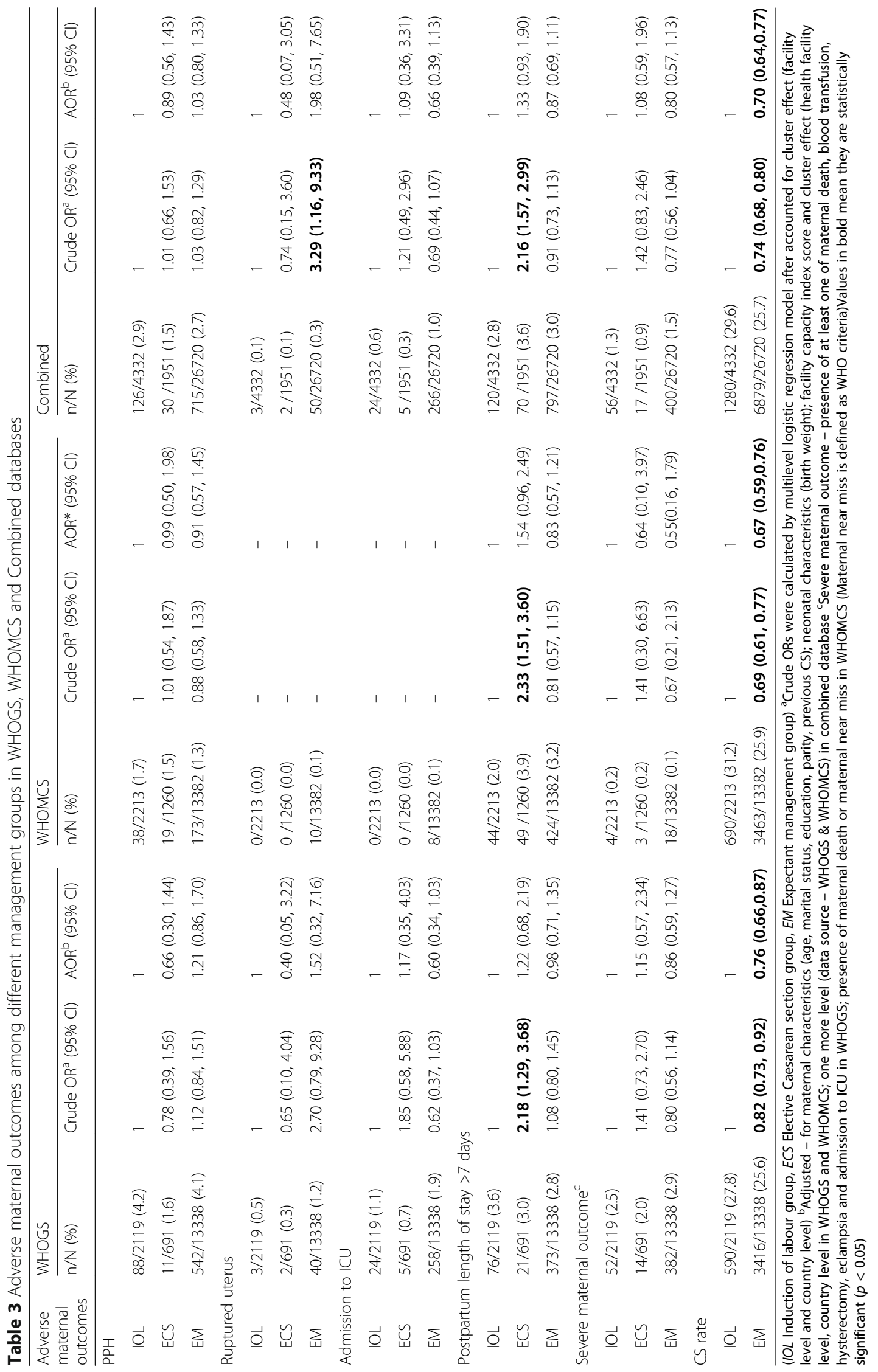




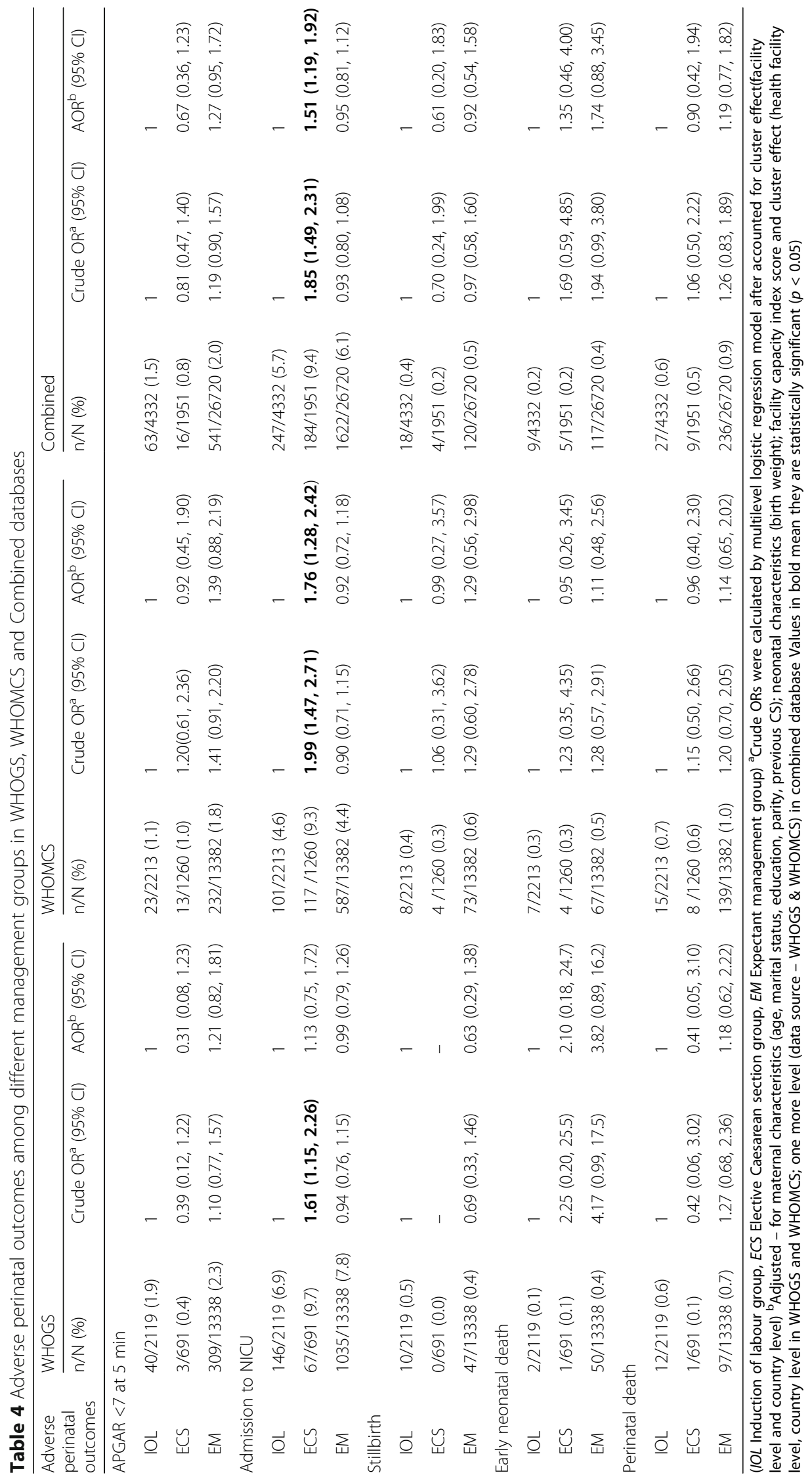


Table 5 Adverse maternal and perinatal outcomes among different management groups in combined databases (Sensitivity analysis)

\begin{tabular}{lll}
\hline Combined & & \\
\cline { 2 - 3 }$/ \mathrm{N}(\%)$ & Crude OR $(95 \% \mathrm{Cl})$ & $\mathrm{AOR}^{\mathrm{b}}(95 \% \mathrm{Cl})$ \\
\hline
\end{tabular}

Adverse maternal outcomes

$\mathrm{PPH}$

$\begin{array}{lll}\text { IOL } & 168 / 5549(3.03) & 1.0 \\ \text { ECS } & 45 / 2493(1.81) & 0.98(0.69,1.40) \\ \text { EM } & 658 / 24,961(2.64) & 0.87(0.71,1.06)\end{array}$

Ruptured uterus

$\begin{array}{lll}\text { IOL } & 5 / 5549(0.14) & 1.0 \\ \text { ECS } & 2 / 2493(0.08) & 0.42(0.09,1.79) \\ \text { EM } & 48 / 24,961(0.31) & 2.72(1.18,6.25)\end{array}$

1.0

$0.90(0.61,1.33)$

$0.88(0.70,1.11)$

Admission to ICU

$\begin{array}{lll}\text { IOL } & 32 / 5549(0.58) & 1.0 \\ \text { ECS } & 9 / 2493(0.36) & 2.06(1.01,4.21) \\ \text { EM } & 254 / 24,961(1.02) & 0.78(0.52,1.15)\end{array}$

Postpartum stay $>7$

$\begin{array}{lll}\text { IOL } & 154 / 5549(2.78) & 1.0 \\ \text { ECS } & 101 / 2493(4.05) & 2.48(1.87,3.29) \\ \text { EM } & 732 / 24,961(2.93) & 0.97(0.79,1.18)\end{array}$

Severe maternal outcome

$\begin{array}{cll}\text { IOL } & 79 / 5549(1.42) & 1.0 \\ \text { ECS } & 24 / 2493(0.96) & 1.50(0.94,2.40) \\ \text { EM } & 370 / 24,961(1.48) & 0.72(0.54,0.94) \\ \text { CS rate } & & \\ \text { IOL } & 1591 / 5549(28.67) & 1.0 \\ \text { EM } & 6026 / 24,961(24.14) & 0.70(0.65,0.75)\end{array}$

Adverse perinatal outcomes

APGAR $<7$ at 5 min

$\begin{array}{lll}\text { IOL } & 109 / 5549(1.97) & 1.0 \\ \text { ECS } & 29 / 2493(1.17) & 0.86(0.56,1.30) \\ \text { EM } & 482 / 24,961(1.94) & 0.88(0.70,1.10)\end{array}$

Admission to NICU

$\begin{array}{lll}\text { IOL } & 344 / 5549(6.22) & 1.0 \\ \text { ECS } & 257 / 2493(10.31) & 1.89(1.57,2.29) \\ \text { EM } & 1452 / 24,961(5.83) & 0.82(0.72,0.94)\end{array}$

Stillbirth

$$
\begin{array}{ll}
\text { IOL } & 25 / 5549(0.45) \\
\text { ECS } & 7 / 2493(0.28) \\
\text { EM } & 110 / 24,961(0.44)
\end{array}
$$

1.0

$0.91(0.40,2.08)$

$0.94(0.60,1.48)$

Early neonatal death

$\begin{array}{lll}\text { IOL } & 23 / 5549(0.42) & 1.0 \\ \text { ECS } & 7 / 2493(0.28) & 0.91(0.39,2.08) \\ \text { EM } & 101 / 24,961(0.41) & 0.93(0.59,1.48)\end{array}$

Perinatal death

$1.56(0.65,3.76)$

$0.72(0.45,1.14)$
Table 5 Adverse maternal and perinatal outcomes among different management groups in combined databases (Sensitivity analysis) (Continued)

\begin{tabular}{llll}
\hline & \multicolumn{3}{l}{ Combined } \\
\cline { 2 - 4 } & $\mathrm{n} / \mathrm{N}(\%)$ & Crude $\mathrm{OR}^{\mathrm{a}}(95 \% \mathrm{Cl})$ & $\mathrm{AOR}^{\mathrm{b}}(95 \% \mathrm{Cl})$ \\
\hline IOL & $48 / 5549(0.87)$ & 1.0 & 1.0 \\
ECS & $14 / 2493(0.56)$ & $0.93(0.51,1.69)$ & $0.75(0.39,1.43)$ \\
EM & $210 / 24,961(0.84)$ & $0.92(0.66,1.27)$ & $0.90(0.63,1.27)$
\end{tabular}

IOL Induction of labour group, ECS Elective Caesarean section group, EM Expectant management group

${ }^{a}$ Crude ORs were calculated by multilevel logistic regression model after accounted for cluster effect(facility level, country level, data source -WHOGS \& WHOMCS)

${ }^{b}$ Adjusted for maternal characteristics (age, marital status, education, parity, previous CS); neonatal characteristics (birth weight); facility capacity index score and cluster effect (facility level, country level, data source - WHOGS \& WHOMCS)

for 44 weeks and aOR18.04; 95\% CI: $1.96-166.11$ for 45 weeks. Details are presented in Table 6.

\section{Discussion}

The prevalence of prolonged pregnancy was about $8 \%$ of total deliveries. The prevalence of prolonged pregnancy varies across the world from 3 to $12 \%$ [25] and our analysis supports those findings. The prevalence is affected by accuracy of gestational age estimation and adoption of elective induction of labour before 41 weeks of gestation [8]. The risk of stillbirth increased significantly when gestational age was 43 weeks or over compared to 41 weeks.

ECS was significantly associated with increased risk of adverse perinatal outcomes compared to IOL for NICU admission in WHOMCS and combined databases. Compared to IOL, EM was significantly associated with decreased risk of CS consistently in all three databases.

Four previous systematic reviews [12, 14-16] reported the effect of IOL compared to EM among prolonged/postterm pregnancies. Systematic reviews of Wennerholm et al., 2009 [15] and Sanchez-Ramos et al., 2003 [16] reported no significant difference in perinatal deaths while those of Gülmezoglu et al., 2012 (Cochrane review) [12] and Hussain et al., 2011 [14] reported significantly lower perinatal death in IOL group. Three systematic reviews i.e. Sanchez-Ramos et al., Wennerholm et al. and Gülmezoglu et al. reported significant lower CS delivery rate in IOL compared to EM. These reviews support the WHO recommendation of routine use of IOL for pregnancies at 41 completed weeks. Our analysis of routinely collected data showed no significant difference in perinatal deaths, however there was a significant lower CS rate in EM compared to IOL in all databases. Possible explanations include: (1) in the Cochrane review, number of perinatal deaths were very small (only 10 deaths) but perinatal death rate of EM was 8 times more than IOL $(0.32 \%$ vs. $0.04 \%)$. In our 
Table 6 Risk of stillbirth by week delivered

\begin{tabular}{llll}
\hline Stillbirth & \multicolumn{1}{l}{ Combined } & & \\
\cline { 2 - 4 } & $\mathrm{n} / \mathrm{N}(\%)$ & Crude OR $(95 \% \mathrm{Cl})$ & $\mathrm{AOR}^{\mathrm{b}}(95 \% \mathrm{Cl})$ \\
\hline 41 completed week & $95 / 25,631(0.37)$ & 1.0 & 1.0 \\
42 completed week & $32 / 6335(0.51)$ & $1.15(0.76,1.71)$ & $1.47(0.82,2.62)$ \\
43 completed week & $10 / 829(1.21)$ & $2.91(1.52,5.58)$ & $3.45(1.32,9.03)$ \\
44 completed week & $3 / 157(1.91)$ & $4.36(1.39,13.64)$ & $6.15(1.38,27.48)$ \\
45 completed week & $2 / 51(3.92)$ & $11.12(2.72,45.41)$ & $18.04(1.96,166.11)$ \\
\hline
\end{tabular}

${ }^{a}$ Crude ORs were calculated by multilevel logistic regression model after accounted for cluster effect (facility level, country level, data source -WHOGS \& WHOMCS) ${ }^{b}$ Adjusted for maternal characteristics (age, marital status, education, parity, previous CS); neonatal characteristics (birth weight); facility capacity index score and cluster effect (facility level, country level, data source - WHOGS \& WHOMCS)

analysis, perinatal death was more common $(272$ deaths) but perinatal death rate of EM was only 1.4 times more than IOL $(0.88 \%$ vs. $0.62 \%)$, (2) our results were from hierarchical databases and we adjusted for multiple potential confounders and clustering effects of facility and country levels in the analysis, this could lead to more conservative confidence interval and (3) the number of participants in the Cochrane review were quite similar across comparison groups but that of our study groups were quite different in number. One more explanation for the difference between our findings and the systematic reviews may relate to difference in context. Trials are generally conducted in highly controlled, often ideal settings (and often in highresource settings), which may favour efficacy. Our data relates more to real-life practices (i.e. effectiveness) in resource-limited settings, where the intervention may not be as beneficial as trials might suggest. A recently published before and after study comparing a policy induction of labour at 41 weeks versus at 42 weeks showed a significantly lower CS rate, 15\% versus $19.4 \%$ ( $p=0.0135)$ in a 41 -week policy. There were no significant differences in maternal or neonatal outcomes [26].

In spite of our extensive literature search, we did not identify any studies comparing IOL and ECS for women at or beyond 41 completed weeks of gestation. Similar with our results, previous study conducted among women at all gestational ages also showed that CS increased the risk of maternal and neonatal adverse outcomes [17].

To our knowledge, this analysis was the first report of the adverse pregnancy outcomes of ECS among women with prolonged pregnancy. This study was based on two large WHO databases conducted in Africa, Asia, Latin America and the Middle East regions. Data were systematically collected by trained personnel. Thus the results of this study reflect the actual practices in participating facilities and their pregnancy outcomes in these facilities.

However, the WHO databases were collected for other specific objectives rather than to explore our study question. Moreover, the two surveys used slightly different case record forms to collect individual and institutional characteristics. Consequently, a few adverse outcomes (postpartum haemorrhage, uterine rupture and severe maternal outcome) had slightly different definitions. Despite this, we elected to combine the databases and analyzed these outcomes collectively as we regarded the outcomes as sufficiently similar.

Both databases were facility based, conducted mainly in larger, secondary and tertiary facilities where CS was available - this might lead to over-representation of adverse outcomes among women and their newborns. Thus, this study results might not be representative in smaller or different facilities. The primary data source was routine hospital records; these may not be ideal in many facility settings. Some facilities encountered suboptimal record collection such as lack of documented diagnosis. It may be due to inability to diagnose condition, failure to recognize condition or failure to document diagnosis, rather than absence of condition. To address this, several facilities adopted the study data collection form as a platform for their medical records. In the WHOMCS, in cases of unclear or missing information, medical staffs were asked to clarify information in the medical record.

The gestational age used was the best obstetric estimate based on local practices. The method of GA assessment was unknown; but usually included the calculation from last menstrual period and/or ultra-sonographic examination. Inaccurate estimate of gestational age can lead to over-estimate prolonged pregnancy [27] and thus may have affected the results of this analysis. However, this misclassification would bias the risk assessment toward unity. The risk that we have estimated should be more conservative. We also tried to minimize the effect of gestational age inaccuracy on this analysis by excluding facilities with GA missing $>5 \%$ and with unreliable information on gestational age distribution such as more than $70 \%$ of all deliveries occurred at a specific week, or where more than $30 \%$ or less than $1 \%$ of all deliveries were preterm (Fig. 1). Furthermore, data concerning 
with methods, types of drugs and routes of administration for IOL cervical ripening methods, oxytocin augmentation protocols, use of partogram, use of electronic fetal monitoring and epidural analgesia rates were not available in databases, so, we could not adjust for these variations to evaluate the outcomes of IOL group.

The differences in institutional intrapartum practices might influence the pregnancy outcomes. We used multilevel logistic regression model to account this effect and hierarchical survey design, using two levels (individual and facility level) for separate analysis of WHOGS and WHOMCS. Moreover, two WHO surveys conducted at different time period and the intrapartum practices of the same facilities might change in two surveys. We accounted for this effect, source of data (WHOGS and WHOMCS) as an additional level for the analysis in combined database.

In addition we did the sensitivity analyses and it showed similar associations among the three management groups when some women were reclassified based on their management beyond 41 weeks of gestation. The other limitation of this study is that it was hospital based and didn't have information on those women who didn't come to the hospital for delivery.

\section{Conclusions}

Compared to IOL, ECS significantly increased risk of NICU admission while EM was significantly associated with decreased risk of CS. ECS should not be recommended for women at 41 completed weeks of pregnancy without spontaneous pain. However, the choice between IOL and EM should be cautiously considered since the available evidences are still quite limited.

\section{Abbreviations}

AIDS: Acquired Immune Deficiency Syndrome; CS: Caesarean Section; ECS: Elective Caesarean Section; EM: Expected Management; GA: Gestational age; HIV: Human Immunodeficiency Virus; IOL: Induction of Labour; UNDP: United Nations Development Programme; UNFPA: United Nations Population Fund; UNICEF: United Nations International Children's Emergency Fund; WHO: World Health Organization; WHOGS: World Health Organization Global Survey; WHOMCS: World Health Organization Multicountry Survey

\begin{abstract}
Acknowledgments
We thank WHO for giving permission to use WHOGS and WHOMCS databases. We would like to thank Dr. Cameron Hurst for giving technical guidance in multilevel modeling. We thank all members of the WHO Multi-Country Survey on Maternal and Newborn Health Research Network, including regional and country coordinators, data collection coordinators, facility coordinators, data collectors, and all staffs of the participating facilities who made the survey possible. This manuscript represents the views of the named authors only, and not the views of their institutions or organizations.
\end{abstract}

\section{Funding}

This secondary analysis study was financially supported by Thailand Research Fund (Distinguished Professor Award). The sponsors had no role in data collection, analysis, or interpretation of the data, the writing of the report, or the decision to submit for publication.

\section{Availability of data and materials}

The datasets generated and/or analyzed during the current study are not publicly available due to they belonged to Department of Reproductive Health and Research, The World Health Organization but could be available from $\mathrm{WHO}$ on reasonable request.

\section{Authors' contributions}

KSM formulated the research question. KSM, ML and PL developed study protocol and analysis plan. KSM cleaned and analyzed the data. KSM, ML and PL drafted the manuscript. All authors reviewed and approved the final version of the manuscript.

\section{Ethics approval and consent to participate}

The WHOGS and WHOMCS were approved by the WHO Ethical Review Committee and the relevant ethical clearance bodies in participating countries and facilities. Written consent from individual women was not needed because there was no contact between the data collectors (who extracted routine medical record data) and individual women, and all data was anonymized.

\section{Consent for publication}

Not applicable.

Competing interests

The authors declared that they have no competing interests.

\section{Publisher's Note}

Springer Nature remains neutral with regard to jurisdictional claims in published maps and institutional affiliations.

\section{Author details}

${ }^{1}$ Department of Epidemiology and Biostatistics, Faculty of Public Health, Khon Kaen University, Khon Kaen 40002, Thailand. Department of Biostatistics, University of Public Health, Yangon, Myanmar. ${ }^{3}$ UNDP/UNFPA/ UNICEF/WHO/World Bank Special Programme of Research, Development and Research Training in Human Reproduction (HRP), Geneva, Switzerland. ${ }^{4}$ Department of Reproductive Health and Research World Health Organization, Geneva, Switzerland. ${ }^{5}$ Department of Obstetrics and Gynecology, University of Campinas, Campinas, Brazil. ${ }^{6}$ Department of Social Medicine, Ribeirão Preto Medical School, University of São Paulo, Ribeirão Preto, SP, Brazil. ${ }^{7}$ Center for Population Health Research, National Institute of Public Health, Cuernavaca, Mexico. ${ }^{8}$ Department of Obstetrics \& Gynecology, Fortis Memorial Research Institute, Gurgaon, India. ${ }^{9}$ Department of Obstetrics and Gynaecology, Faculty of Medicine, Khon Kaen University, Khon Kaen 40002, Thailand.

Received: 6 April 2017 Accepted: 10 October 2017

Published online: 30 October 2017

\section{References}

1. WHO. World Health Organization recommendations for induction of Labour 2011. http://apps.who.int//iris/handle/10665/44531. Accessed 24 Aug 2014.

2. Martin JA, Hamilton BE, Ventura SJ. Et al.National Vital Statistics Reports. Report no. 1.Hyattsville, Maryland. United States of America: National Vital Statistics System; 2012

3. The Health and Social Care Information Centre.NHS Maternity Statistics 2011-2012 Summary report. Geneva, Switzerland: National Health Service; 2012. http://www.hscic.gov.uk/hes.

4. Li Z,Zeki R, Hilder $L$, et al.Australian institute of health and welfare. Australia's mothers and babies 2010.Canberra,ACT,Australia:Australianlnstitute ofHealth and welfare; 2012. http://www.aihw.gov.au/publication-detail/?id= 60129542376.

5. Vogel JP, Souza JP, Gülmezoglu AM. Patterns and outcomes of induction of labour in Africa and Asia: a secondary analysis of the WHO global survey on maternal and neonatal health. PLoS One. 2013;8(6):e65612.

6. Guerra G, Cecatti J, Souza J, Faúndes A, Morais S, Gülmezoglu A, et al. Factors and outcomes associated with the induction of labour in Latin America. BJOG Int J Obstet Gynaecol. 2009;116(13):1762-72.

7. Chantry AA, Lopez E. Fetal and neonatal complications related to prolonged pregnancy. J Gynécologie Obstétrique. Biol Reprod. 2011;40(8):717-25. 
8. Mandruzzato G, Alfirevic Z, Chervenak F, Gruenebaum A, Heimstad R, Heinonen $\mathrm{S}$, et al. Guidelines for the management of postterm pregnancy. J Perinat Med. 2010;38(2):111-9.

9. Roos N, Sahlin L, Ekman-Ordeberg G, Kieler H, Stephansson O. Maternal risk factors for postterm pregnancy and cesarean delivery following labor induction. Acta Obstet Gynecol Scand. 2010;89(8):1003-10.

10. Vayssière C, Haumonte J-B, Chantry A, Coatleven F, Debord MP, Gomez C, et al. Prolonged and post-term pregnancies: guidelines for clinical practice from the French College of Gynecologists and Obstetricians (CNGOF). Eur J Obstet Gynecol Reprod Biol. 2013;169(1):10-6.

11. Paterson-Brown S. Should doctors perform an elective caesarean section on request? BMJ. 1998;317(7156):462-5.

12. Gülmezoglu AM, Crowther CA, Middleton P, Heatley E. Induction of labour for improving birth outcomes for women at or beyond term. Cochrane Database Syst Rev2012;6:CD004945.

13. Caughey AB, Sundaram V. Systematic review: elective induction of labor versus expectant management of pregnancy. Ann Intern Med. 2009;151(4): 252-63. W53-63

14. Hussain AA, Yakoob MY, Imdad A, Bhutta ZA. Elective induction for pregnancies at or beyond 41 weeks of gestation and its impact on stillbirths: a systematic review with meta-analysis. BMC Public Health. 2011; 11(Suppl 3):S5

15. Wennerholm U-B, Hagberg H, Brorsson B, Bergh C. Induction of labor versus expectant management for post-date pregnancy: is there sufficient evidence for a change in clinical practice? Acta Obstet Gynecol Scand. 2009;88(1):6-17.

16. Sanchez-Ramos L, Olivier F, Delke I, Kaunitz AM. Labor induction versus expectant management for postterm pregnancies: a systematic review with meta-analysis. Obstet Gynecol. 2003;101(6):1312-8,

17. Lumbiganon P, Laopaiboon M, Gülmezoglu AM, Souza JP, Taneepanichskul $\mathrm{S}$, Ruyan $\mathrm{P}$, et al. Method of delivery and pregnancy outcomes in Asia: the WHO global survey on maternal and perinatal health 2007-08. Lancet. 2010; 375(9713):490-9.

18. Souza JP, Gülmezoglu AM, Vogel J, Carroli G, Lumbiganon P, Qureshi Z, et al. Moving beyond essential interventions for reduction of maternal mortality (the WHO multicountry survey on maternal and newborn health): a cross-sectional study. Lancet. 2013;381:1747-55.

19. How does the World Bank classify countries? - World Bank Data Help Desk https://datahelpdesk.worldbank.org/knowledgebase/articles/378834. Accessed 12 Oct 2016.

20. Souza JP, Gülmezoglu AM, Carroli G, Lumbiganon P, Qureshi Z, WHOMCS Research Group. The world health organization multicountry survey on maternal and newborn health: study protocol. BMC Health Serv Res. 2011;11:286.

21. Karlsen S, Say L, Souza J-P, Hogue CJ, Calles DL, Gülmezoglu AM, et al. The relationship between maternal education and mortality among women giving birth in health care institutions: analysis of the cross sectional WHO global survey on maternal and perinatal health. BMC Public Health 2011;11(1):1-10.

22. Vogel J, Souza J, Mori R, Morisaki N, Lumbiganon P, Laopaiboon M, et al. Maternal complications and perinatal mortality: findings of the World Health Organization multicountry survey on maternal and newborn health. BJOG Int J Obstet Gynaecol. 2014;121:76-88.

23. Bates D, Maechler M, Bolker B, Walker S. Ime4: linear mixed-effects models using Eigen and S4 [internet]. R package version. 2014;1:1-7. http://CRAN.Rproject.org/package=Ime4

24. Core Team R. R: a language and environment for statistical computing [internet]. Vienna, Austria: R foundation for statistical. Computing. 2014; http://www.R-project.org/

25. Caughey AB, Chelmow D. Postterm pregnancy. 2013http:// emedicinemedscapecom/article/261369-overviewAccessed 12 Aug 2014.

26. Bleicher I, Vitner D, lofe A, Sagi S, Bader D, Gonen R. When should pregnancies that extended beyond term be induced? J Matern Fetal Neonatal Med. 2017:30(2)

27. Galal M, Symonds I, Murray H, Petraglia F, Smith R. Postterm pregnancy. Facts, Views \& Vision in Obstetric and Gynaecology. 2012;4(3):175-87. http:// www.ncbi.nlm.nih.gov/pmc/articles/PMC3991404/

\section{Submit your next manuscript to BioMed Central and we will help you at every step:}

- We accept pre-submission inquiries

- Our selector tool helps you to find the most relevant journal

- We provide round the clock customer support

- Convenient online submission

- Thorough peer review

- Inclusion in PubMed and all major indexing services

- Maximum visibility for your research

Submit your manuscript at www.biomedcentral.com/submit
Biomed Central 